Surprisingly in 53\% of NDBE tissue samples we identified clonal expansion of cells ( $>10 \%$ mutant fraction) harbouring mutations in one or more of $13 / 15$ of these putative driver genes. No difference in the frequency of mutation of these genes was observed between any of the disease stages studied. TP53 mutations clearly delineate between HGD/OAC and benign NDBE $(\mathrm{p}<0.001)$. Whilst SMAD4 mutations are only observed in OAC $(\mathrm{p}<0.001)$ demonstrating for the first time a clear genetic difference between the two.

Conclusion Mutagenic processes active in OAC are also active in the earliest stages of BE. Recurrent driver mutations identified in cancer may be acquired very early in the disease and may provide little or no progression advantage. Molecular diagnostic approaches must account for this.

Disclosure of Interest None Declared.

\section{PTU-154 DIFFERENCES IN COELIAC SEROLOGY OUTCOMES APPEAR DEPENDANT UPON THE ORIGIN OF THE REQUEST: A SEVEN YEAR REVIEW OF ANTI-TISSUE TRANSGLUTAMINASE TESTING}

K Bundhoo*, D Housley, D Gordon, K Rostami, M Johnson. Gastroenterology, Luton and Dunstable Hospital, Luton, UK

\subsection{6/gutjnl-2014-307263.228}

Introduction Coeliac disease is the most common genetically based food intolerance worldwide with a UK prevalence of 1 in 150. The condition poses a diagnostic difficulty due to the often non-specific symptoms at presentation and a sub-group who will be asymptomatic. Successful diagnosis is based upon a high degree of suspicion, correct screening and a subsequent confirmatory test with an intestinal biopsy. Serological testing via antiendomysial antibody (AEA) and anti-tissue transglutaminase (ATTG) is a simple, highly accurate method of screening, with a sensitivity and specificity in the region of $95-98 \%$ and $95-97 \%$, respectively. Due to these attributes, serological screening accounts for a significant proportion of laboratory workloads nationwide.

Methods All ATTG coeliac serology requests made in a busy district general hospital over a 7 year period between 2007 and 2013 were reviewed, with a particular focus upon the positive results. A further assessment of case records and in particular the origin of requester was conducted. A positive result was issued by the laboratory if the ATTG was greater than or equal to 4 .

Results Overall, a total of 29795 ATTG requests were made to the biochemistry department, of which results were obtained from 28819. Of this number, 1005 were performed in inpatients, 7140 in outpatients and 20674 were from primary care. In the GP cohort, 785/20674 (3.8\%) proved to be positive. In comparison $37 / 1005(3.7 \%)$ were positive from the inpatient group, and 371/7140 (5.2\%) were positive from the outpatient cohort. The deficit in results gained was primarily due to the rejection of samples by the laboratory as being "not indicated" in 402 inpatient cases (27\%) and 4 outpatient cases. No GP requests were rejected. The overall numbers of ATTG requests also increased year on year with 913 being performed in 2008 and 6483 in 2013.

Conclusion It is clear the demand for coeliac serology is increasing with its use becoming more widespread in a variety of clinical settings. The mere fact that patients are presenting to hospitals for outpatient appointments or inpatient assessments, places them in a self selecting group where one would expect to see a higher frequency of positive coeliac serology. This was duly noted in our outpatient cohort, but the inpatients had a similar positive pick up rate to the GP cohort. It is possible this result was slightly skewed by the high rejection rate seen with inpatient requests. The high positive pick up rate from specialist outpatient clinics emphasises the importance of having a high degree of clinical suspicion in order to make an appropriate diagnosis.

Disclosure of Interest None Declared.

\section{PTU-155 PROGRESSION OF LOW GRADE DYSPLASIA TO HIGH GRADE DYSPLASIA IN BARRETT'S OESOPHAGUS IN A SINGLE CENTRE}

${ }^{1}$ L-L Clark*, ${ }^{2} \mathrm{G}$ Richardson, ${ }^{2} \mathrm{~S}$ Paterson. ${ }^{1}$ Gastroenterology, Victoria Infirmary Glasgow, Glasgow, UK; 'astroenterology, Forth Valley Royal Hospital, Larbert, UK

\subsection{6/gutjpl-2014-307263.229}

Introduction Barrett's oesophagus is a common condition found in $4 \%$ of patients undergoing upper gastrointestinal endoscopy. ${ }^{(1)}$ The association between Barrett's oesophagus and oesophageal adenocarcinoma has been well established. Scotland has a particularly high incidence of both Barrett's and adenocarcinoma of the oesophagus.

The risk of progression from high grade dysplasia (HGD) to oesophageal adenocarcinoma (OAC) has been reported at approximately $10 \%$ per year. ${ }^{1}$ However the risk of progression from low grade dysplasia (LGD) is harder to quantify with studies showing progression to OAC from $0.6-1.69 \%$ per year, not dissimilar to that of non-dysplasic Barrett's. ${ }^{2}$ More recently the SURF trail reported a $25 \%(17 / 68)$ progression from LGD to $\mathrm{HGD} / \mathrm{OAC}$ in a surveillance group with LGD versus radiofrequency ablation. ${ }^{3}$

Methods In 2009 a clinical database of Barrett's patients was developed in Forth Valley Hospital to ensure appropriate surveillance as per BSG guidelines. All patients diagnosed with Barrett's were cared for by one responsible team, a dedicated Barrett's endoscopy list was developed, and the use of narrow band imaging was introduced. At the end of 2012 the database was interrogated to assess the progress of all patients who had been diagnosed with LGD within the previous three years.

Results There were 915 patients with Barrett's on the database, of which 829 were under follow up. 85 (10\%) had LGD and of this patient cohort 19 had progressed to HGD. The progression rate from LGD to HGD was 22\% (19/85). The median follow up of patients with LGD was 29 months (range 12-34 months). All patients who progressed from LGD to HGD had endoscopic therapy with endoscopic mucosal resection and /or ablative therapies. There are no recorded cases of progression of LGD to OAC.

Conclusion The progression rate from LGD to HGD is similar to reported rates found in the SURF trial. This suggests that LGD carries a greater risk of progression, and therefore worse prognosis than previously reported. This is a potential group of patients in whom to consider early intervention rather than adopt the standard surveillance strategy. Further studies to evaluate the effectiveness of treatment rather than surveillance in this group should be considered.

\section{REFERENCES}

1 Consensus Statements for Management of Barrett's Dysplasia and Early-Stage Esophageal Adenocarcinoma, Based on a Delphi Process. Gastroenterology 2012;143 (2):336-346

2 Sachin W, Sharad M. How to manage a Barrett's Esophagus patient with low grade dysplasia. Clin Gastroenterol Hepatol. 2009;7(1):27-32 